\title{
III VirginiaTech Development of a Low-Flying CubeSat for F-Region Characterization
}

Anthony Wolosik ${ }^{1}$, Dr. Jonathan Black ${ }^{2}$, Dr. Gregory Earle ${ }^{3}$, and Dr. Robert McGwier ${ }^{4}$

${ }^{1}$ Graduate Student, Department of Aerospace and Ocean Engineering, Virginia Tech

${ }^{2}$ Director, Aerospace Systems Laboratory, Hume Center for National Security and Technology, Associate Professor, Aerospace and Ocean Engineering, Virginia Tech

${ }^{3}$ Professor, Department of Electrical and Computer Engineering, Virginia Tech

${ }^{4}$ Research Professor and Director of Research, Hume Center for National Security and Technology, Virginia Tech

\section{Introduction}

Plasma irregularities in the F-region $(200-800 \mathrm{~km})$ of the night-time equatorial ionosphere have been shown to significantly influence the performance and reliability of space borne and ground based radio communication systems. Equatorial Spread-F (ESF) episodes may cause the disruption of satellite operations, communications, and navigation due to backscatter and radar scintillation. Current records of rocket, satellite, and radar data are not of sufficient quantity to allow for accurate models and predictions of the spectrum of irregularities. To provide a better priction of the spection pediclions of the spectrum of inegut commenicalime measurnents in atellite orbits at altitudes in the bottomside $\mathrm{F}$ region have a very limited lifetime due to atmospheric drag. The objective of the Low-Flyer CubeSat mission is to provide scientific data through improved in situ instruments and methods to better characterize the F-region of the ionosphere. Achieving low altitudes in the bottomside $\mathrm{F}$ region while still enduring a mission lifetime that encompasses each season, the Low-Flyer CubeSat mission intends to achieve these goals through the use of on board propulsion and an increased ballistic coefficient. This presentation will overview the technical details from current projects and that will allow for a successful mission of the Low-Flyer CubeSat.

\section{Next Generation LAICE Instrumentation}

The Lower Atmosphere/Ionosphere Coupling Experiment (LAICE) is a $6 \mathrm{U}$ CubeSat mission focused on gravity wave propagation from low altitudes into the ionosphere. Among the various scientific instruments incorporated to successfully achieve LAICE's mission goals is the Retarding Potentia Analyzer (RPA). The RPA is a forward velocity direction, or ram, in situ instrument that is designed to provide information on the state of ionospheric plasma, including the plasma density, ion temperature, ion velocity, and the ratio of light to heavy ions. The Low-Flyer mission uses the RPA design from the Lower Atmosphere/lonosphere Coupling Experiment (LAICE) mission as a baseline.

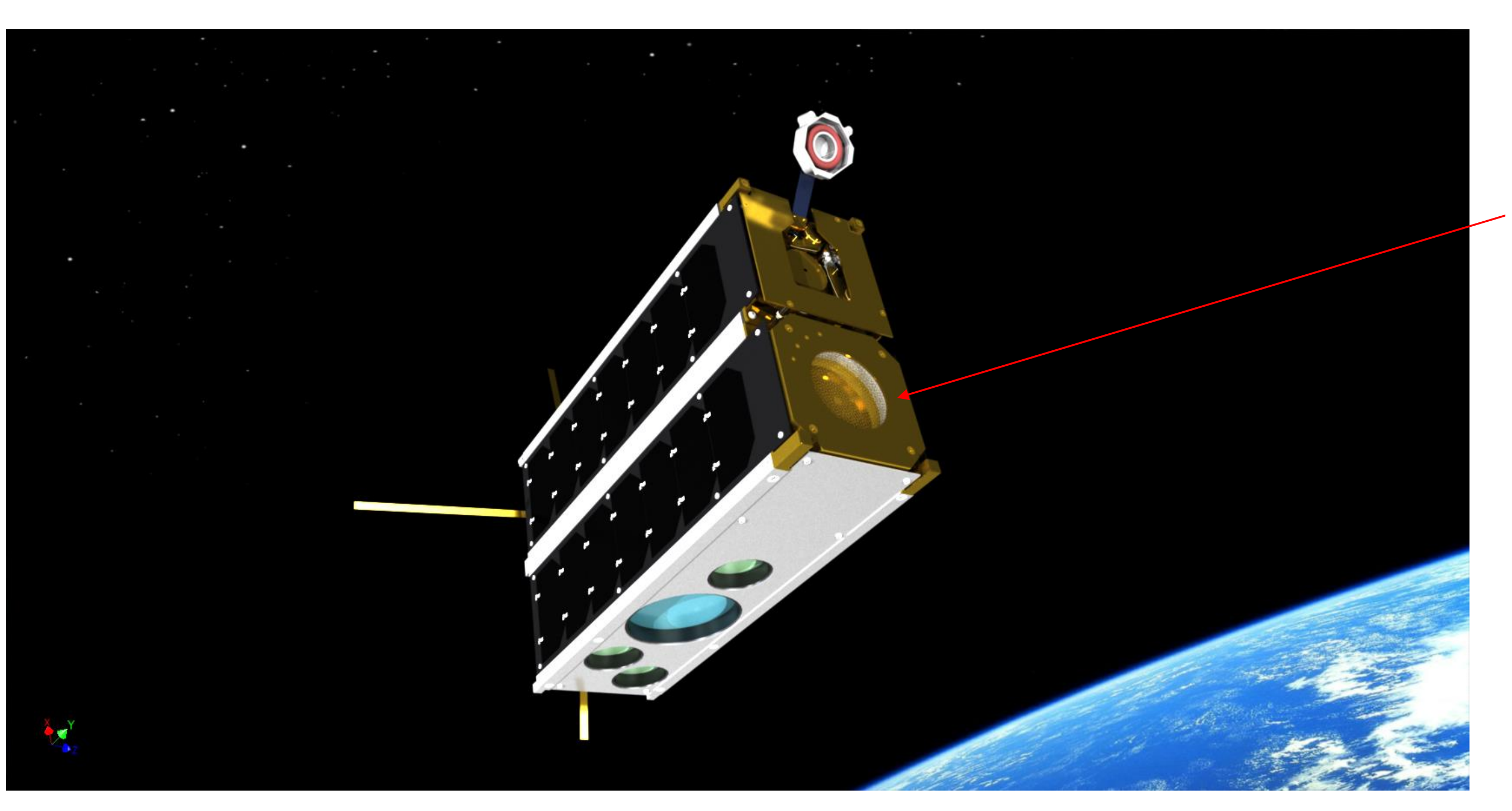

Figure 1 - Cartoon view of the LAICE satellite, with ram-facing RPA shown above. Image used with permission from Dr. Greg Earle.
Phase 4B / GEO EMCOMM Mission

\section{Instrumentation}

Virginia Tech, the Radio Amateur Satellite Corporation (AMSAT), and our partners are collaborating to give amateur radio operators critical new capabilities in disaster situations. The objective of this mission is to place an amateur radio payload in geosynchronous orbit, enabling resilient 24/7 coverage for amateur radio communications. Trained American Radio Relay League (ARRL) operators mobilize to disaster areas to provide emergency communications for first responders. This experiment will test the capability of using satellite communications in disaster relief situations. The amateur radio payload will include a reprogrammable software-defined radio (SDR) an launch as a hosted payload. After successful implementation of the SDR provided by Rincon Research Corporation on the GEO Mission, simila implementation will be used on the Low-Flyer in order to achieve it measurements of over the horizon tada communications.

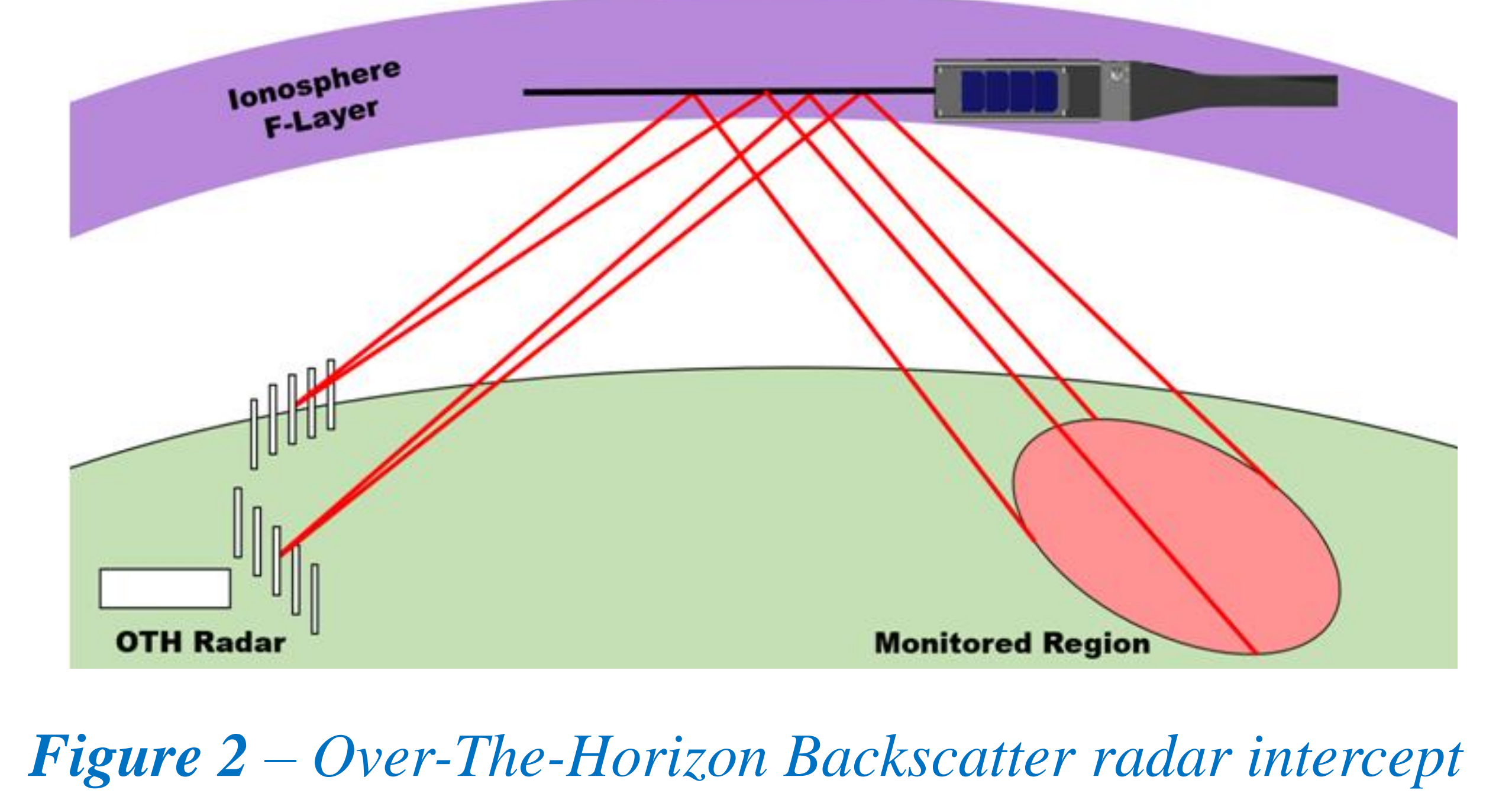

profile example for the Low-Flyer mission.

\section{Testing Facilities}

The utilization of in-house testing facilities will allow for rapid end-to-end testing of the Low-Flyer CubeSat and help identify potential risks that typically go unseen in CubeSat development. The Virginia Tech CubeSat Attitude Control Simulator (CSACS), designed and fabricated at Virginia Tech's Space Systems Laboratory, is an air-bearing platform used to test various CubeSat attitude determination and control systems. Coupled with the development of a Helmholtz cage, realistic simulations of on-orbit CubeSat performance will be capable at Virginia Tech.
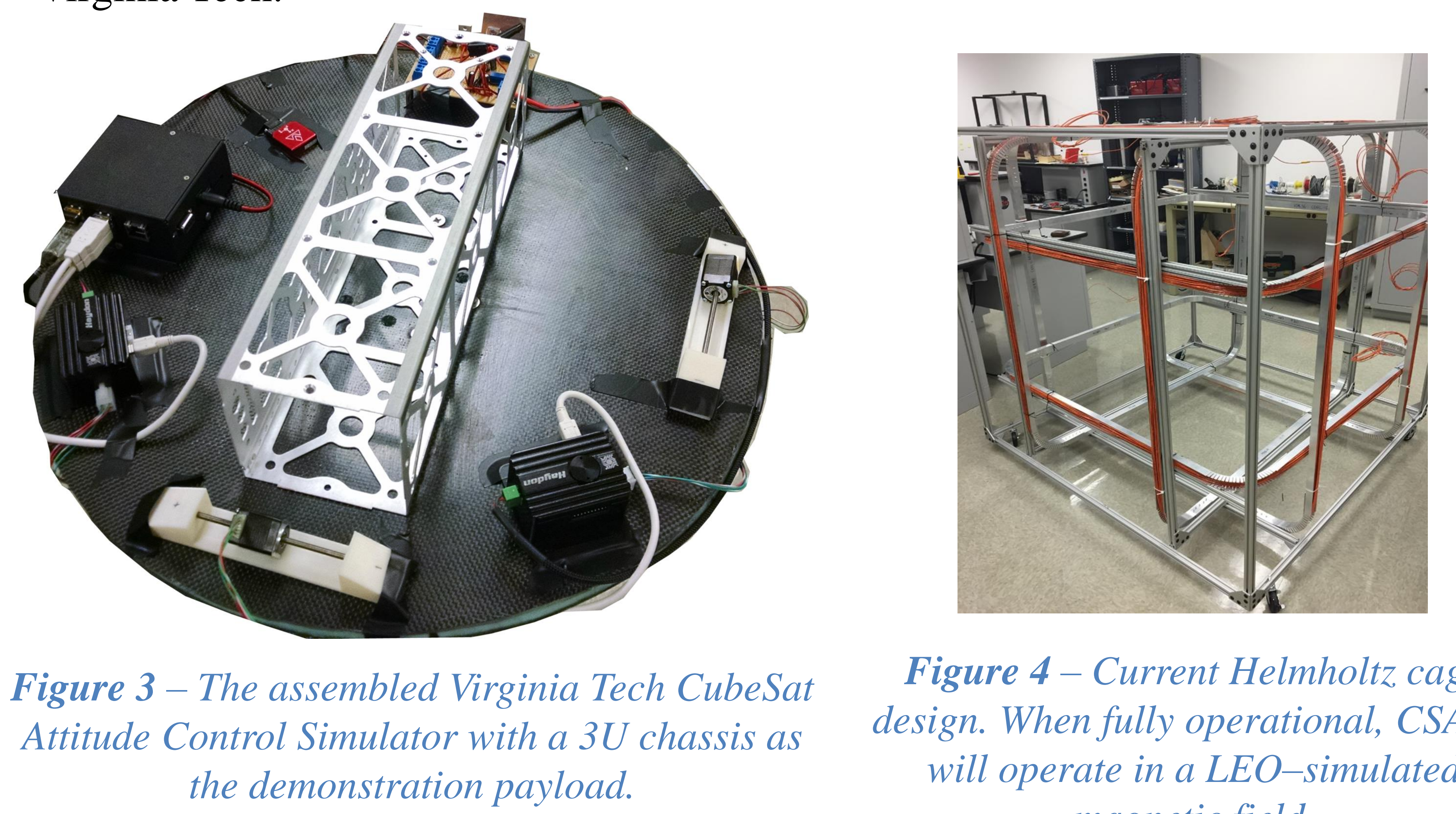

Figure 4 - Current Helmholtz cage
design. When fully operational, CSACS will operate in a LEO-simulated magnetic field.

\section{Low-Flyer Mission Description}

The Low-Flyer is a proposed 6U low-altitude satellite that will implemen in-house instrumentation to be flown at altitudes routinely considered too low for satellite missions (200-300 km). Its' unique tapered design raises the ballistic coefficient which greatly increases the mission duration when compared to a conventional CubeSat. After insertion, the Low-Flyer will naturally decay to the lower limits of the scientific data region and then mplement a propulsive thrust to increase its' orbit and again naturally decay. The additional satellite lifetime that results from the increased ballistic coefficient and propulsive maneuver allows for maximum time spent in the scientific regions of the mission.

The goals of the mission are to:

. Utilize a next generation RPA design on the ram face of the satellite to make continuous in situ measurements of plasma and neutral density fluctuations and ion temperature in the lower F-Region of the ionosphere.

2. Since the desired altitude for taking RPA measurements puts the LowFlyer at an altitude where radio waves tend to deflect in the ionosphere, implement a deployable, high frequency (HF) antenna from the rear end of the spacecraft to intercept over-the-horizon (OTH) radar waves. Refer to Figure 2



Figure 5 - The Low-Flyer is separated into a Rear Frame and Forward

\section{Conclusions}

To provide a better understanding and predict F-region phenomena that may disrupt communications, it is important to perform in situ, or local, real-time measurements in areas that these irregularities begin. Typically, satellite orbits at altitudes in the bottomside $\mathrm{F}$ region have a limited lifetime due to atmospheric drag. The Low-Flyer CubeSat mission intends to implement a unique tapered design, coupled with propulsion, that enables it to achieve both low altitudes in the bottomside $\mathrm{F}$ region while still enduring a mission lifetime that encompasses each season.

\section{References}

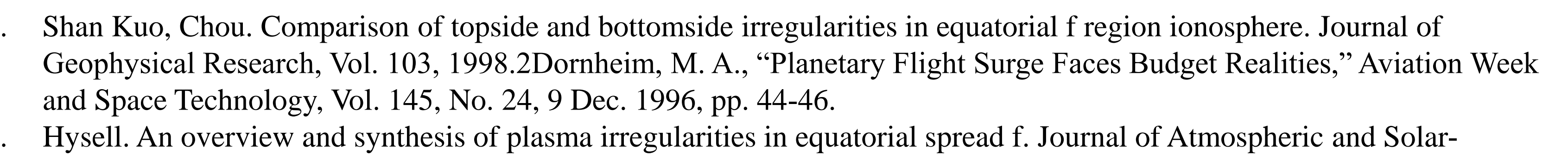

